\title{
THE IMPORTANCE OF THE MECHANICAL FACTOR IN THE TREATMENT OF PULMONARY TUBERCULOSIS
}

\author{
By H. MORRISTON DAVIES \\ (From University College Hospital and the City of London Hospital for \\ Diseases of the Chest)
}

\author{
With Plates 12 and 13
}

\section{Introduction.}

IF a large series of cases of pulmonary tuberculosis be studied clinically, radiographically, and from the point of view of their reaction to treatment, it is surprising how rare it is to find any two of them which show any uniform similarity. Yet, despite this fact, there seems to be a tendeney at the present time to classify the types of disease under a very few brief headings, and to distribute the patients for the purposes of treatment into as small a series of groups as possible. This method has had a considerable degree of success since the aim and object in each of the groups is, in a great measure, to increase the physical fitness of the patient and his capacity, therefore, to react to the disease. The success in the treatment has not, however, been as great as it is possible to obtain because the individuality of the case has been obscured by the group system, and because also the mechanical changes in the lungs and in the thorax have not been given sufficient attention. It is in this respect particularly that the individuality of the patient exists. It is true that in a very small proportion of cases the mechanical factor is dealt with, and collapse of the lung is obtained by introducing nitrogen into the pleural eavity; but even the rationale of this treatment is not always recognized, as is shown by the prejudice which still exists against it, and by the opinion which is still too often expressed that the treatment should not be tried except as a 'last resort'.

There are four factors which are of the utmost importance when determining the course of treatment most suitable for a patient suffering from pulmonary tuberculosis. They are:

1. The capacity of the patient's tissues to react against the disease.

2. The extent to which this reaction can be increased by hygienic measures, exercises, and so forth.

3. The extent and character of the mechanical changes which have already taken place.

[Q. J. MI., April, 1918.] 
4. The presence of accessory foci of chronic septic absorption (especially those of the nose, mouth, and pharynx).

It is on these four factors that the prognosis also must be based.

I do not intend to deal here with the first two and the last of these, but wish to discuss the mechanical changes that may occur and the various ways in which it is possible to overcome to a greater or less extent the symptoms they give rise to and the disadvantageous influence which these changes may in their turn exert on the course of the primary disease. It must, however, be clearly borne in mind that though I deal with the one factor as separate from the other three, yet in the actual treatment of a case it is of little use to treat the mechanical changes unless every available means is taken at the same time to maintain and to increase the general tone of the body.

l'he mechanical disabilities are due primarily to the anatomical and physiological conditions of the lungs and thorax, and secondarily to the fibrotic changes in the lung parenchyma, to the occasional formation of cavities, and to the interpleural adhesions which develop as a result of active or quiet (so far as symptoms are concerned) pleurisies. These disabilities are found in all chronic diseases of the lung, but are most evident in tuberculosis and bronchiectasis.

The primary causes are too well known to need more than brief reference. The lungs are enclosed in air-tight cavities bounded by semi-rigid walls and are kept in a state of expansion by a constant negative pressure. Diminution in the size of the lung must be accompanied, therefore, by an increase in the negative pressure in the pleural cavities, or by the yielding of one or more of the sides of the cavities. When the diminution in the volume of the lung is slowly progressive, it is rare to find an increase in the negative pressure equal to 2 or $3 \mathrm{~mm}$. of mercury. The walls bounding the lung yield with difficulty; the mediastinum is the least rigid and the ribs the most, whilst the displacement of the diaphragm is dependent to a great extent on the presence or absence of direct pull on that muscle through adhesions. In children the flexibility of all the walls is considerably greater than in adults and diminishes steadily with increase in years.

In chronic pulmonary diseases there is always the formation of abnormal fibrous tissue due to the reaction in the lung to the irritation set up by the disease. The fibrous tissue contracts and there is, therefore, a progressive diminution in the volume of the lung. This exerts a constant traction on the surrounding walls, which in turn resist the process. There are thus two opposing forces at work, each interfering with the function of the other. On the one hand, there is the resistance offered to the contraction of the lung by the surrounding walls and by the intrapleural negative pressure; on the other hand, there is the constant pull of the fibrous tissue, exerted either through the negative pressure or by direct pull by adhesions on the thorax, on the mediastinum, and on the diaphragm limiting the normal movements of these parts and causing deformity.

The intrapulmonary fibrosis has also a striking influence on the bronchi, 
giving rise to the most characteristic secondary changes associated with pulmonary tuberculosis and to a group of symptoms which are more difficult to treat even than those of the initial lesion. The morbid anatomy change is directly or indirectly a bronchial dilatation. The strands of fibrous tissue which radiate from the bronchi to the periphery are, as I have said, constantly contracting. If there was no resistance at the outer surface of the lung, the greater part of the force of this contraction would be exerted in collapsing the lung. Owing, however, to the resistance offered by the surrounding walls, the force of the contracting fibrous tissue is exerted on the walls of the bronchi as well as on the walls of the pleural cavity, and a direct bronchial dilatation ensues. When, however, the fibrosis is massive in character, there is strangulation of the larger bronchi : this leads to obstruction of the air entry into those areas of the lung supplied by the affected tubes, and later to a secondary bronchiectasis. The dilated bronchi are also sooner or later infected by saprophytic and pyogenic organisms.

The formation of fibrous tissue continues so long as there is any active tuberculous disease or secondary infection, either of intrapulmonary cavities or of dilated bronchial tubes. As regards the latter, the fibrosis which is responsible for the secondary infection is also aggravated by it and a vicious circle is established.

The fibrosis is the attempt on the part of the tissues to check the spread of the disease and to repair the damage which has been done by it. It may be successful so long as the process bas not spread beyond the capacity of the surrounding structures to compensate for the contraction. If the disease and the associated fibrosis are of greater extent, then the usefulness of this new tissue formation is limited, is frustrated, or is even turned to disadvantage by the normal anatomical and physiological conditions.

A cavity in the lung differs very little from a chronic abscess in any other part of the body. If a bronchus affords free drainage of the cavity it remains comparatively dry and innocuous. But if, on the other hand, the drainage is inadequate on account either of the size or the position of the opening, then there is retention of secretions, secondary infection, toxic absorption, and the contained pus is usually offensive.

No deep-seated chronic abscess can heal while its walls are separated. Healing is difficult, even under the most advantageous circumstances, when the cavity is infected by tubercular as well as by pyogenic organisms. The approximation of the walls of a moderate-sized or Jarge cavity in the lung must necessarily be a practical impossibility without adventitious assistance, unless the thoracic walls are extremely plastic. The most that can be done without surgical intervention is temporarily to reduce the infection in the cavity and possibly to effect some slight reduction in size by encouraging the free expansion of the more normal parts of the lung. So long as the cavity persists, however, it is always liable at any time to become re-infected.

The formation of interpleural adhesions is not necessarily preceded by a painful pleurisy. Nor does a painful pleurisy always imply the subsequent 
adherence of the pleural membranes. The existence of adhesions enables the lung to exert direct traction on the surrounding walls. The effect of this on the diaphragm is to produce fixation and at times striking distortion of that muscle. One may infer from this that the distortion of the chest-wall is greater when adhesions are present than when the contraction of the lung exerts its influence indirectly (i.e. through the intrapleural negative pressure). Dense adhesions on the mediastinal aspect fix the mediastinum and so limit the displacement of the contained structures.

It is difficult to determine the precise extent to which these abnormal mechanical conditions may be responsible for symptoms, may interfere with treatinent, and may affect the prognosis of the ease. Their adverse influence is in certain respects unquestionable, but in others it is more problematical. These mechanical conditions are without doubt responsible for the secondary changes which occur in the bronchial tubes, changes which are accompanied by symptoms of chronic bronchitis or of secondary infection. They are the main factor in the causation of shortness of breath, in that the free expansion of the lung is inhibited either by the direct contraction and resistance of the strands of fibrous tissue, or by the obstruction of free air entry into the alveoli by massive fibrosis round the bronchial tubes; thus interfering with the correlated rhythm of the lung and of the thorax during respiratory movements. They are responsible, in certain cases, for the retention of secretions, and they interfere with the free blood supply and lymph flow in the affected area or areas of the lung. They may be the determining factor in the formation of cavities; they certainly prevent their collapse, thereby interfering with the healing processes and encouraging the occurrence of secondary infections and of ulceration of the exposed vessels. Lastly, when the visceral and diaphragmatic pleural membranes are adherent, the contraction of the fibrous tissue leads to distortion of the diaphragm, which change is at times accompanied by a constant and extremely distressing dry cough.

The contraction of the chest-wall, the displacement of the mediastinum and its contents, and the compensatory emphysema of the unaffected lung areas are also the product of the diminution in the volume of the lung due to the fibrosis. These changes are the effort on the part of nature to counteract the abnormal mechanical factors. In the earliest stage of the disease they may be actually sufficient to produce adequate compensation for the mechanical disabilities. An early case of pulmonary tuberculosis may in fact be defined as one in which the changes in the surrounding structures (i.e. the contraction of the walls and the emphysema) have been able to keep pace with the fibrous contraction of the diseased part of the lung: in other words, they have been able up till then to compensate for the mechanical disabilities. As the disease progresses, the extent of this natural compensation is increasingly limited, and later is totally inadequate. The secondary changes in the lung now manifest themselves, and they may eventually become the predominating feature. Once the pathological changes have exceeded the limits of easy compensation it must necessarily follow that 
the success which can be obtained by methods of treatment, which do not include the means of artificially compensating for or of overcoming the mechanical disabilities, must be limited or must fail.

The rationale of all modern surgical intervention for the treatment of pulmonary tuberculosis is the readjustment of the normal, physiological, and anatomical conditions of the lung and of the walls surrounding that organ, so as to accommodate them to and compensate for the mechanical disabilities due to the various pathological changes which have taken place in the lung and in the pleural cavity. The operations are designed to remove those disabilities which are an almost insuperable bar to the successful treatment of the patient by hygienic or other medical forms of treatment. But each of these surgical measures possesses other advantages: they have a direct beneficial effect on the disease, on the symptoms of the disease, on the secondary complications, and, therefore, on the general health of the patient and his power of resistance to the invasion of the tubercle bacilli. They do not all of them shorten the time during which the patient has to receive treatment, but they very materially lessen the period during which he has to abandon all work and to devote himself exclusively to the task of attempting to combat the primary disease and its complications,

\section{The Methods of Operative Intervention.}

Four methods are available, ${ }^{1}$ namely: 1. Displacement of the lung by nitrogen (artificial pneumothorax). 2. Local displacement of the lung and of the parietal pleura by foreign substances. 3. Displacement of the whole lung and of the chest-wall by rib mobilization. 4. Paralysis of the diaphragm by section of the phrenic nerve.

1. Nitrogen displacement. In a normal person it is possible to obtain complete collapse of the whole of one lung by injecting nitrogen into the pleural cavity. The lung is collapsed round its root; there is no longer a negative intrapleural pressure, and the respiratory movements of the chest-wall and of the diaphragm exert comparatively little influence on the Jung. In a patient suffering from pulmonary tuberculosis, the completeness of the collapse of the lung may be interfered with by intrapleural adhesions or by intrapulmonary changes such as massive fibrosis and pneumonic consolidation. The limitation in the extent of the collapse due to the intrapulmonary changes is only very rarely sufficient to affect adversely the advantages which may be expected from this method of treatment. Interpleural adhesions, on the other hand, may be so widespread as altogether to prevent the introduction of gas, or by their extent or character they may seriously limit the displacement of the organ. In the absence of these complications the collapse of the lung, which can be obtained by displacement by nitrogen, offers a comparatively simple and extremely satisfactery

1 The treatment of ingravescent pneumothorax due to spontaneous rupture of the lung is not included here, but will be discussed later. 
means of compensating for the mechanical disabilities brought about by the disease.

The presence of gas at atmospheric or at a positive pressure in the pleural cavity removes that constant tension on the surfaces of the lung which interferes with the contraction of the fibrous tissue. This is now able freely to contract without dragging on the walls of the bronchi. Cavities due either to dilated bronchi or to disintegration of the lung parenchyma become obliterated and their walls approximated ; there is no longer, therefore, retention of secretions, and consequently the absorption of the toxins due to the secondary infections is greatly diminished and the danger of a haemorrhage by ulceration into an artery is abolished. The symptoms also associated with these pathological changes-pyrexia, cough, sputum-diminish in a striking fashion or disappear. The lung which responded inadequately to the enlargement of the chest during inspiration, owing to the resistance of the fibrous tissue, is now placed at rest, and the shortness of breath is consequently diminished. The patient's general condition improves immediately with the diminution of the symptoms, with the absence of septic intoxication, with the improvement in the night sleep and of the appetite. The ability to do an increasing amount of work without aggravation of the symptoms leads to a steady improvement of the power of resistance to the primary infection.

I have already, in other papers, described the technique of nitrogen displacement, ${ }^{2}$ and there is, therefore, no necessity to describe this again. Recent experience has, however, led me to modify my opinion on three points. During this last summer I have started twelve new cases, and only one of these patients have I kept rigidly confined to bed during the preliminary injections. After the first two injections the patient has remained in bed for forty-eight hours so that his reaction to the treatment could be exactly estimated. After the subsequent injections the patient has been allowed to get up in from twelvo to twenty-four hours unless there were any special symptoms which contraindicated this course. Whereas previously some 3,500 to 4,000 c.c. of nitrogen were required to bring about complete displacement of the lung, in all but one of the last series practically twice as much gas was required. The exception was the patient who was kept in bed during the whole period of time of induction of the artificial pneumothorax on account of tachycardia, the result of prolonged septic intoxication. This increase in the amount of gas required is due to the exercise promoting more rapid absorption.

The period of six weeks which I have formerly advocated as being the maximum time which should elapse between the first few refills is, I am now certain, too long an interval. For the first six months the refills should be done at intervals of not more than a month.

It is usually stated that about 33 per cent. of patients treated by nitrogen displacement develop some fluid in the pleural cavity. In none of the cases above mentioned did an effusion occur, and in one patient only of the previous

2 British Medical Journal, March, 1914 ; Choyce's System of Surgery. 
series. ${ }^{3} \quad$ An effusion may be the result of trauma to the pleura, or of rupture of adhesions, but $I$ believe that in the large majority of cases it is an indication of a mild infection.

All patients who are being treated by nitrogen displacement should be examined periodically with the $\mathrm{X}$-rays. This is the only certain means of ascertaining the manner and extent of the displacement and the position and character of any adhesions which may be present. It is by means of the X-rays only that it is possible to determine when the collapse of the lung is complete. Neither the amount of gas run in, nor the pressure of that gas, nor the displacement of the heart affords reliable evidence.' The displacement of the heart depends largely on the mobility of the mediastinum. A positive pressure may indicate complete collapse of the lung, but it may equally well be present when the displacement is only partial, and is then due to adhesions or to the resistance offered by the intrapulmonary fibrosis or consolidation.

In comparatively few cases of pulmonary tuberculosis are the pleural membranes completely free of adhesions: these were present in every one of the twelve cases of my last series. The character of the adhesions varies greatly : they may be almost universal, and the treatment is then impossible; they may be few in number but dense in character, or multiple but slender. The treatment in every case in which adhesions are present must vary with the character of them, and this can only be determined with the X-rays. When the upper lobe is adherent to the dome of the pleural cavity and the remainder is free, the whole organ is displaced in an upward and inward direction, and the collapse is often as efficient, though not as complete, as in a case free of all intrapleural fibrosis. ${ }^{4}$

Fixation of the lung to the diaphragm by a basal pleurisy is often an annoying complication, as with collapse of the lung there is constant traction on and displacement of the muscle. This at times is comparatively symptomless, but usually there is a dry irritating cough which may be paroxysmal in character when the patient exerts himself, or it may be the constant accompaniment of all actions such as laughing, sighing, or the taking of a deep breath. This symptom lessens as the gas in the pleural cavity is absorbed and the lung re-expands, but with each refill it occurs with the same irritating persistence. In all cases in which the basal adhesions are directly interfering with the treatment, it is advisable to paralyse that side of the diaphragm by section of the phrenic nerve in the neck. The results obtained by this treatment will be considered later.

In one case in which the characteristic symptoms due to traction on the diaphragm developed as a result of nitrogen displacement, I paralysed the

3 All these patients were examined constantly by the X-rays.

4 In one of my cases in which this condition was present, the displacement of the lung into the upper part of the pleural cavity was immediately effectual in reducing to normal the temperature, which had shown a nocturnal rise of 2 or 3 degrees for several months. There was a corresponding abatement in all the other symptoms. 
phrenic nerve by the injection of absolute alcohol. Although I was successful, it is not a mothod that $I$ would advocate unless the patient refuses operative treatment. The chances of satisfactorily infiltrating the nerve, using a small quantity of alcohol only, are uncertain, owing to the difficulty of gauging the exact thickness of the overlying structures.

The systematic radiographic examination of all cases of tuberculosis undergoing nitrogen displacoment will show the greatest variation in the number, the thickness, and the position of adhesions.

A broad dense adhesion will yield very little to the constant displacement of the lung by nitrogen. The medium-sized adhesions will stretch; some of them sufficiently to enable full displacement of the lung to be obtained. The finest of all will rupture, at times with even a quite small intrapleural positive pressure. The actual separation of adhesions occurs probably during an act of coughing and may be accompanied by a slight momentary pain.

Adhesions which interfere with efficient collapse of the lung, but which allow sufficient displacement to enable the character and extent of the bands to be recognized by radiography, can be dealt with in three ways: $(a)$ By rupture; (b) by stretching; (c) by division.

(a) Rupture. This treatment is justifiable only when there are multiple strands traversing the pleural cavity, and when rupture can be effected by raising the intrapleural tension by a pressure of gas equal to not more than $20 \mathrm{~mm}$. of mercury. I have on one occasion raised the intrapleural pressure for this purpose until it equalled $32 \mathrm{~mm}$. of mercury; but in this case there was a profusion of strands traversing the space between the lung and the chestwall, and the condition of the patient was hopeless unless a considerable displacement of the lung could be effected. The radiogram showed, moreover, that the visceral pleura was less likely to yield than a number of the adhesions: in order to minimize the risk of a pleural reflex, one-third of a grain of morphia had been given previously. The patient describes the sensation, at the moment of rupture, as of something giving way inside the chest; the manometer shows an abrupt fall in the pressure and an increase in the respiratory variations.

(b) Stretching. Medium-sized interpleural bands may be stretched by maintaining a fairly steady positive pressure (equal to about 8 or $10 \mathrm{~mm}$. of mercury) for a considerable period of time (two or three months). Refills are necessary during this period every eight to ten days. By this means I have succeeded in getting almost complete displacement, nearly doubling the amount of the collapse which was at first permitted by the resistance of the adhesions.

The constant stretching of adhesions by pressure is, as a rule, symptomless. Occasionally however, and more particularly if the peripheral end of the adhesion is attached to the diaphragm, slight pain is experienced. Rupture of adhesions may cause a slight pleural effusion; stretching practically never does.

Neither of these two methods of treatment is justifiable unless the processes can be continually controlled by radiography. It may almost be said that it is dangerous to inject gas into the pleural cavity once the intrapleural 
tension is positive, unless an accurate knowledge of the intrapleural conditions has been obtained by the X-rays. One case will help to emphasize this point. A positive pleural pressure had been reached, but it was obvious from the amount of gas which had been run in that the displacement of the ling was incomplete. Radiographic examination revealed that there was a single stout adhesion running from the surface of the lung to the pleura; the central end was situated over a superficial cavity the outer wall of which had become coneshaped from the drag of the fibrous strand. A further increase of intrapleural pressure would have, in all probability, resulted in the rupture of the outer wall of the cavity. Such an adhesion cannot be treated by stretching, but by division only.

(c) Division. The presence of a single or even of two or three interpleural bands, which allow a partial collapse of the lung only but which will not yield further to the constant influence of an intrapleural positive tension, must not be permitted to interfere with the success of the treatment by nitrogen displacement. Such adhesions are usually attached to the lung opposite the area most affected by the disease, and consequently that part of the organ which should be the most collapsed is the least displaced. There is one situation in which a single band-like adhesion is found with great frequency. The central end is attached to the lung near the lower border of the upper lobe, whilst the peripheral end is fixed to the parietal membrane opposite the second rib in the front of the chest, at the side, or posteriorly. The band may be narrow and cylindrical or broad and flat; the latter is more resistant to stretching than the former. If a radiogram shows that such free bands of adhesions are the sole cause of preventing further collapse, and if at the end of a month it is clear that they will not yield to the stretching force applied, they should be divided. Before such an operation is undertaken the direction of the adhesion and the points of attachment must be determined as accurately as possible. The method of approach and of division varies with the type of the band. Those which are over one inch in length are most satisfactorily divided by a special tenotome passed through the chest-wall, the field of operation being rendered visible by $\mathrm{X}$-ray illumination. When the adhesion is short and broad it is not suitable for this method of division, and access must be obtained by opening the chest at a point as close to the band as possible. This section by direct division is rarely indicated, but division by what may be termed indirect vision (X-ray illumination) is much more frequently necessary. The following are the steps of operation :

The day or two before, an injection of gas should be given so as to tighten the band by displacing the lung as far as it will go. Two hypodermic injections, each of a quarter of a grain of morphia, are given, the one half an hour and the other immediately before the operation. The patient lies on a couch with the X-ray tube beneath, while an assistant holds the screen. The tube should be fitted with a diaphragm so as to limit the rays to the field of operation. It is advisable for the surgeon to wear smoked glasses, which are removed only 
when the $\mathrm{X}$-rays are turned on. When the peripheral attachment of the adhesion is to the front of the chest, the tenotome is passed into the pleural cavity through an intercostal space in the mid-axillary line, but if the adhesion is fixed to the lateral or posterior aspect, the tenotome is passed through the front of the chest.

The tenotome which $I$ had made for this purpose has the blade $2 \mathrm{~cm}$. long by $0.6 \mathrm{~cm}$. broad, sharpened at both edges, and gradually increasing in thickness to the shank. This part of the instrument is $12.5 \mathrm{~cm}$. long, and has the same breadth and thickness as the base of the knife, but the edges are rounded. It is in turn attached to a short handle. The shank passes readily through the incision in the chest-wall made by the blade of the tenotome, but fits so closely into it that there is no escape of air. The wound through the pleura closes on withdrawing the knife, and unless there is an immediate fit of coughing, there is no subsequent escape of gas into the cellular tissues. The track through which the tenotome passes is previously anaesthetized with novocaine.

As soon as the tenotome is in the pleural cavity, the room is darkened and the X-rays are turned on. Both the adhesion and the tenotome are now visible, and the latter is directed towards the former till the edge of the knife rests on the band. The resistance which this offers to the knife can be felt and the movement of the band and of the lung produced by the pressure of the knife can be seen. The adhesion is cut through by sawing movements.

A little blood may escape from the cut edges if the adhesion is a broad one, but the bleeding soon stops and is of no material consequence. The patient is kept quite quiet for the next twenty-four hours, but beyond this no special after-treatment is necessary.

2. Local displacement of the lung and of the parietal pleura by foreign substances. This form of treatment is strongly advocated by Tuffier. It is applicable only to those cases in which the disease is limited to one lobe and in which nitrogen displacement is impracticable. Advantage is taken of the fact that even when the two pleural membranes are firmly united by the products of inflammatory action, it is still possible to strip the parietal membrane from off the deep surface of the chest-wall. Such displacement cannot be maintained by injections of gas, as there is no limiting membrane on the outer surface to prevent the escape of gas into the cellular tissues. It is for this reason that the extrapleural space is filled up by some solid substance. The foreign substances used are of two characters: (1) Paraffin, or pastes of which paraffin is the basis; (2) transplanted living tissues, e.g. lipomata, omentum, fibromata. The best results have been obtained with the living tissues.

The indications for this treatment are that the disease is localized, is complicated by considerable mechanical changes, and that the pleural membranes are adherent. It is obvious that such cases are comparatively infrequent.

3. Displacement of the whole lung and of the chest-wall by rib mobilization. In every case in which collapse of the lung by nitrogen displacement has been 
tried but has failed owing to the density of the adhesions, the advisability of obtaining collapse of the lung together with the chest-wall by rib mobilization must be considered. Rib mobilization places the whole lung permanently out of action, and there is no possibility of allowing re-expansion of that organ should there be spread of the disease to the opposite side. In the cases selected for nitrogen displacement, the presence of some disease in the second lung is not necessarily any bar to this method of treatment. But when determining on the suitability of a case for rib mobilization, the presence of any active tubercular disease whatsoever in the opposite lung should always negative the operation. A small quantity of fibrosis is not necessarily a contra-indication. Myocarditis and a rapid pulse due to prolonged septic intoxication are usually improved when collapse can be obtained by nitrogen, but these conditions must be regarded very seriously when considering the question of rib mobilization. It is only a few of those cases, therefore, in which treatment by nitrogen has been tried and failed that are suitable for rib mobilization. The number is still further reduced by the exclusion of those in which the disease is localized to a part only of one lung and in which treatment by nitrogen has been advised because the lesion is progressive or remains chronic despite hygienic measures, or because it has been desirable to enable the patient to resume his occupation at the earliest moment possible. For such localized disease rib mobilization may occasionally be necessary, but a much longer period of time should be allowed for the trial of other and less drastic measures.

In suitable cases-that is, in those in which there is a chronic unilateral tubercular fibrosis and especially in those in which the primary disease is complicated by secondary infection, by cavity formation, and by bronchial dilatation (Plate 12)-rib mobilization will yield most gratifying results. In many respects it is a much more efficient method of treatment than nitrogen displacement because the collapse of the lung once obtained is constant and is permanent. The operation is one, however, which must not be undertaken lightly, or until the fullest examination has been made and consideration given to all the aspects of the case. But the mortality is low and the end results are now sufficiently established to necessitate a more universal recognition of this method of treatment for pulmonary tuberculosis.

I have given a full description of the technique in the British Journal of Surgery, vol. ii, No. 8, 1915. ${ }^{5}$

4. Paralysis of the diaphragm by section of the phrenic nerve. The object of this operation is, equally with the preceding, to compensate for the mechanical disabilities due to the intrathoracic changes produced by pulmonary tuberculosis. The treatment differs, however, from those above described, in that it has very little direct effect on the course of the primary disease; its value is as an adjunct to other forms of treatment by relieving certain distressing symptoms, and by preventing the establishment or the spread of some of the most serious complications.

- 'The Operation of Rib Mobilization for the Treatment of Phthisis.'

Q 2 
I have already called attention to the advantages gained by paralysing the diaphragm when the pull of the lung on that muscle through adhesions, as the result of the collapse of the lung by nitrogen, produces a constant cough. In some cases of extensive fibrosis there is striking distortion of the diaphragm independent of any displacement of the lung by surgical intervention. A 'diaphragmatic' cough is an occasional accompaniment of this change, and complete relief can be afforded by section of the phrenic nerve.

The phrenic is readily accessible for division in the neck immediately above the clavicle, where it lies on the scalenus anticus muscle under cover of the sterno-mastoid. The operation is done under local analgesia. The resulting paralysis of the corresponding half of the diaphragm is, as a rule, complete. That part of the muscle relaxes, and with the $\mathrm{X}$-rays the arch is seen to occupy a position about one inch higher than normal and during quiet respiration to be stationary. During deep inspiration the paralysed half of the muscle rises still higher into the thorax. Very occasionally a considerable portion of the nerve fibres for the diaphragm are conveyed to the phrenic by way of the nerve to the subclavius muscle. Section of the phrenic in the neck is then followed by a paresis only of the corresponding half of the diaphragm (Plate 13).

Since paralysis of the diaphragm is accompanied by an upward displacement of that muscle, it is obvious that there must be a corresponding diminution in size of the base of the pleural cavity and of the lung; and in addition considerable diminution of expansion of the lower lobe during inspiration, as this depends mainly on the downward pull of the diaphragin. The collapse of the base of the lung is not sufficient to make the benefits immediately apparent, but the diminution in the volume of the lobe and the removal of the constant drag on the fibrous tissue must very materially compensate for the existing, and check the further development of the mechanical disabilities. Paralysis of the diaphragm may therefore be regarded as a prophylactic measure against the production or the increase of bronchial dilatation in the lower lobe. ${ }^{6}$

There are thus four separate methods of combating and of compensating for a whole or a part of the mechanical disabilities which develop during the progress of or during the processes of reaction to the disease in cases of pulmonary tuberculosis. Of these, the displacement of the lung by nitrogen is the method of choice in all cases in which it can be carried out. It is without danger, if suitable precautions are taken; the technique is extremely simple in straightforward cases, but considerable experience is required to obtain the best results, since the conditions vary so in every case that it may truly be said that each patient requires individual treatment. The patient is not necessarily confined to bed during the treatment, and the period of his convalescence is greatly reduced. The displacement can be maintained for as long or as short a time as

6. It is for this reason that I advocate section of the phrenic nerve in cases of chronic interstitial basal pneumonia, since these cases, if left untreated, almost invariably develop progressive bronchial dilatation. Influenzal pneumonia is the commonest precursor of bronchiectasis. 
required. When the collapse of the lung is complete, the symptoms disappear rapidly and usually completely, if the disease is unilateral; they are greatly reduced in bilateral cases. The lung is at rest and the primary disease is placed in those circumstances most antagonistic to the spread of the tubercle bacilli.? The secondary infections are reduced by the obliteration of those spaces which favoured the development of them; the danger of secondary haemorrhage is abolished; the tibrosis is no longer interfered with by extra-pulmonary conditions, and is in turn not able to exert its harmful influence on the intrapulmonary tissues. As the result of these changes, the general health of the patient improves with increase in appetite and better sleep. The increase in tone implies an increase in capacity to react against the disease, not only in the side under treatment but in the opposite lung also. Finally, it is possible for these patients to return to their work without danger to themselves or to the community after a period of time which is very greatly less than if the displacement had not been established.

The presence of adhesions necessarily limits the rapidity and possibly also the extent of the improvements. For this reason the stretching or the division of adhesions is always indicated when practicable.

Collapse of the lung by rib mobilization has the same effect upon the disease and its complications as has collapse by nitrogen displacement, but the operation is more drastic and the displacement is permanent. The number of suitable cases is, therefore, very limited, since the presence of active disease in the opposite lung is a necessary bar to this treatment.

Cases suitable for local replacement of part of the lung and of the parietal plenra are also few in number. There must necessarily also bo a considerable difficulty in obtaining a mass of living tissue sufficient in size to effect the required degree of displacement.

Lastly, paralysis of the diaphragm has a direct beneficial effect on basal tuberculosis. The indirect value of it is considerable both as a check to the diaphragmatic type of cough and as a prophylactic measure against the increase of secondary bronchiectasis and the infection of the lower lobes.

Reference has been made previously to the cases of pulmonary tuberculosis in which the fibrosis is massive in character, resulting in obstruction in the air entry into the alveoli and later extensive bronchial dilatation. This type in its earlier stages will improve under treatment by nitrogen displacement; but in the later ones, when the bronchial changes are advanced, more satisfactory results are obtained by rib mobilization.

The fibrous tissue changes in the lung which persist after arrest of the disease are of a different character to those present whilst the pathological processes are in an active state. With cessation of the irritation due to the presence of disease, there is progressive modification and refinement of the masses of fibrous tissue. There is always a scar to show where the disease has

7 This part of the subject I have dealt with in another paper, "The Necessity for the Fuller' Consideration of the Local Processes of Disease and Repair', to be published shortly. 
been, but the coarse fibrous bundles become gradually absorbed and replaced by fine radiating strands which have comparatively little power to produce further morbid changes.

During the spring and early summer of this year (1917) I have had the opportunity of examining and of treating with Dr. Kincaid some eighty patients suffering from pulmonary tuberculosis. As a result of the presence of mechanical disabilities, nitrogen displacement was attempted in twelve cases; in three of these it was abandoned owing to the extensiveness of the intrapleural fibrosis. Most of the remaining nine had some form or another of adhesions. In one, fine strands were ruptured. In three, adhesions were stretched by maintaining a constant intrapleural positive tension. In one, an adhesion was divided; in one, the diaphragm was paralysed by the injection of alcohol into the phrenic nerve to abolish the symptoms due to the pull on the muscle by diaphragmatic adhesions. In all nine cases there was marked diminution of the symptoms and a corresponding improvement in the general condition.

In two patients, in whom there was considerable secondary bronchiectatic change in one lower lobe, the diaphragm was paralysed by section of the phrenic nerve. In one patient, who developed a pleural effusion, the fluid was replaced by oxygen and the displacement of the lung was subsequently maintained by nitrogen. Treatment also was necessary in one case in which a spontaneous ingravescent pneumothorax developed.

Brief allusion must be made to the treatment of this condition. If the symptoms are urgent and threatening the life of the patient, instant relief can be obtained by passing a large-sized hypodermic needle through the chest-wall. This needle is fixed in position by thread and strapping, and the outer end should be covered with a few layers of sterilized gauze.

When the symptoms are less acute it is possible, while allowing for the escape of the excess of air in the pleural cavity, to maintain a constant slight positive intrapleural pressure. This is particularly desirable as, unless the opening in the lung closes soon after the rupture and is prevented from being repeatedly reopened by the exertion of coughing, there is the danger of a permanent fistula resulting. The method of regulating the pressure is as follows:

If the needle which passes through the chest-wall is connected by tubing to a Wolff's bottle partly filled with water, the depth of the opening of the tubing below the surface of the fluid can be altered so that the gas will escape whenever the intrapleural pressure exceeds the required. maximum; this should be equal to about $5 \mathrm{~mm}$. of mercury. It will be found, however, that a cough raises the pressure considerably above this. With each cough there will be an escape of gas, and if the opening in the lung has closed, the intrapleural pressure will become progressively less positive and then more negative. To obviate this, the Wolff's bottle must be replaced by a horizontal spiral of some four turns of narrow glass tubing, the distal end of which opens into the base of a broad cylindrical receiver. The spiral coils should be $1 \frac{1}{2}$ in. in diameter. The receiver must be broad, so that when the fluid is displaced into it from the 
THE TREATMENT OF PULMONARY TUBERCULOSIS

219

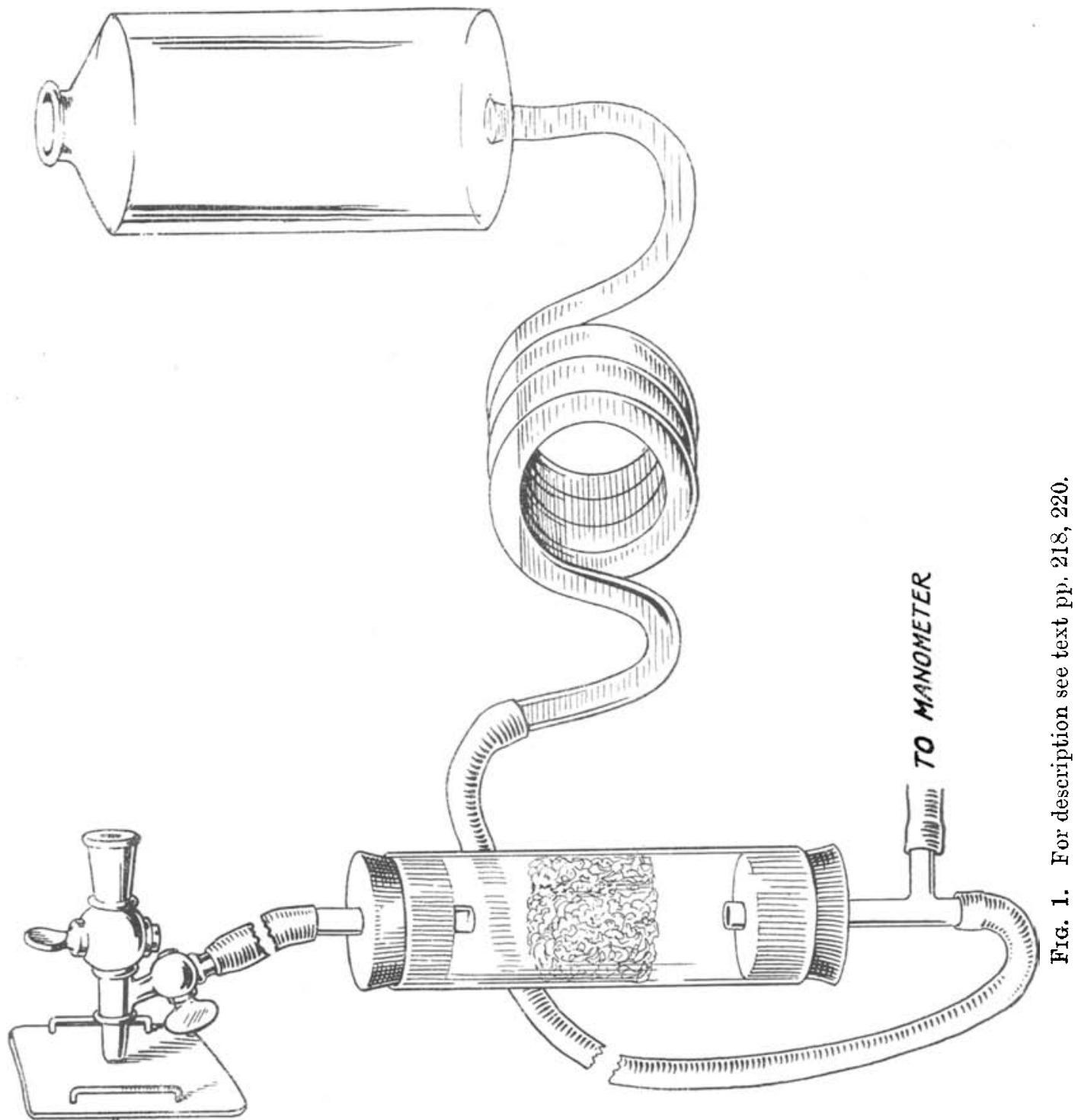


spiral tubing the depth of water is not increased more than half an inch. Above, it is in direct communication with the open air; the spiral and receiver must be below the level of the patient. The intrapleural pressure is regulated by the extent to which these are filled with water. The water in the succession of loops of tubing prevents the escape of gas when the pressure in the pleural cavity is momentarily, even though considerably, raised by acts such as sneezing and coughing. When, however, there is a steady rise of pressure, the water is displaced from the spiral into the receiver and gas escapes until the intrapleural pressure is again equal to the height of the column of water (Fig. 1).

In conclusion, a word must be said on the advisability of carrying out the operative treatment for pulmonary tuberculosis in sanatoria as opposed to hospitals or nursing homes. A considerable experience of both enables me to assert without hesitation the desirability of the former, provided always that the necessary facilities are available. The results obtained in hospitals and nursing homes may be extremely gratifying, but one is often hampered by the inability to give to the patient those advantages which are to be obtained in a fully equipped sanatorium only. Nitrogen displacement need scarcely interfere with the special routine of sanatorium life, and if the $X$-rays are available, as they always should be, the treatment can be carried out under complete control.

The time required for the operations and for the immediate convalescence when rib mobilization is done is about eight weeks. It is an inestimable advantage to the patient if that time can be spent away from the stagnant and depressing atmosphere of a town, in a sanatorium where the air is fresher, the outlook more extensive, and the patient, when sufficiently recovered, can be wheeled from bis room into the grounds. For it to be possible for these operations to be done in a sanatorium, however, the place must be properly equipped and nur'ses competent for the care of surgical cases must be available.

At present the importance, and even I may say the necessity, for the closest alliance between surgery and medicine in the treatment of pulmonary tuberculosis has not been appreciated. When this comes to pass, the equipment of a modern sanatorium will include facilities for radiography and for operative treatment. 


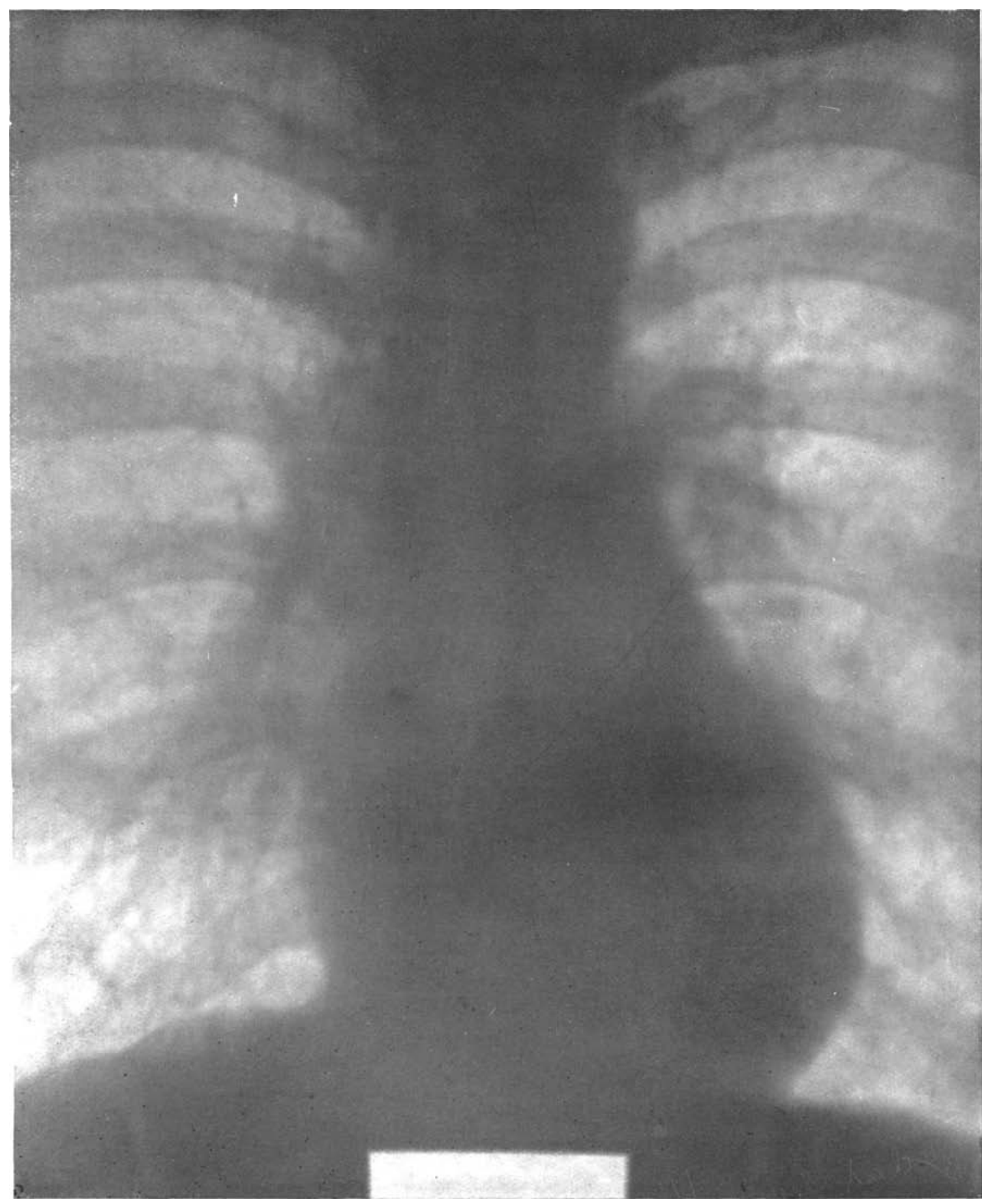

Bronchial dilatation of the right lower lobe (compare with Plate 13). 


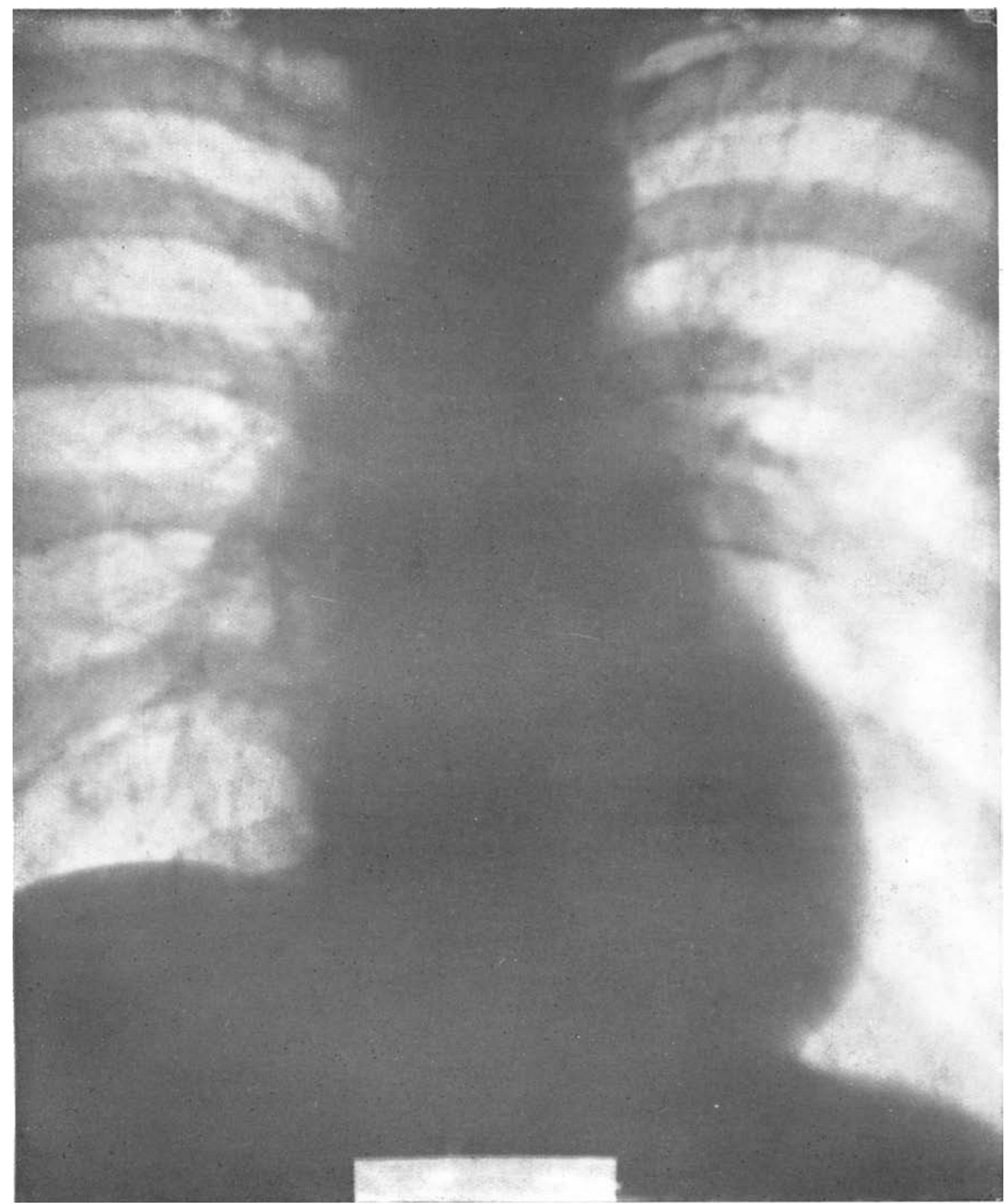

Same case as shown in Plate 12. Paralysis of the right dome of the diaphrigm by division of the phrenic nerve. 